\title{
Highlights from the High Altitude Water Cherenkov Observatory
}

\author{
John Pretz ${ }^{* a}$ for the HAWC Collaboration ${ }^{b}$ \\ ${ }^{a}$ Department of Physics, Pennsylvania State University, State College, PA, USA \\ ${ }^{b}$ For a complete author list, see www.hawc-observatory.org/collaboration/icrc2015.php \\ Email: pretz@psu.edu
}

The High Altitude Water Cherenkov (HAWC) Gamma-Ray Observatory was completed this year at a 4100-meter site on the flank of the Sierra Negra volcano in Mexico. HAWC is a water Cherenkov ground array with the capability to distinguish $100 \mathrm{GeV}-100 \mathrm{TeV}$ gamma rays from the hadronic cosmic-ray background. HAWC is uniquely suited to study extremely high energy cosmic-ray sources, search for regions of extended gamma-ray emission, and to identify transient gamma-ray phenomena. HAWC will play a key role in triggering multi-wavelength and multimessenger studies of active galaxies, gamma-ray bursts, supernova remnants and pulsar wind nebulae. Observation of $\mathrm{TeV}$ photons also provide unique tests for a number of fundamental physics phenomena including dark matter annihilation and primordial black hole evaporation. Operation began mid-2013 with the partially-completed detector. Multi-TeV emission from the Galactic Plane is clearly seen in the first year of operation, confirming a number of known $\mathrm{TeV}$ sources, and a number of AGN have been observed. We discuss the science of HAWC, summarize the status of the experiment, and highlight first results from analysis of the data.

The 34th International Cosmic Ray Conference,

30 July- 6 August, 2015

The Hague, The Netherlands

\footnotetext{
* Speaker.
} 


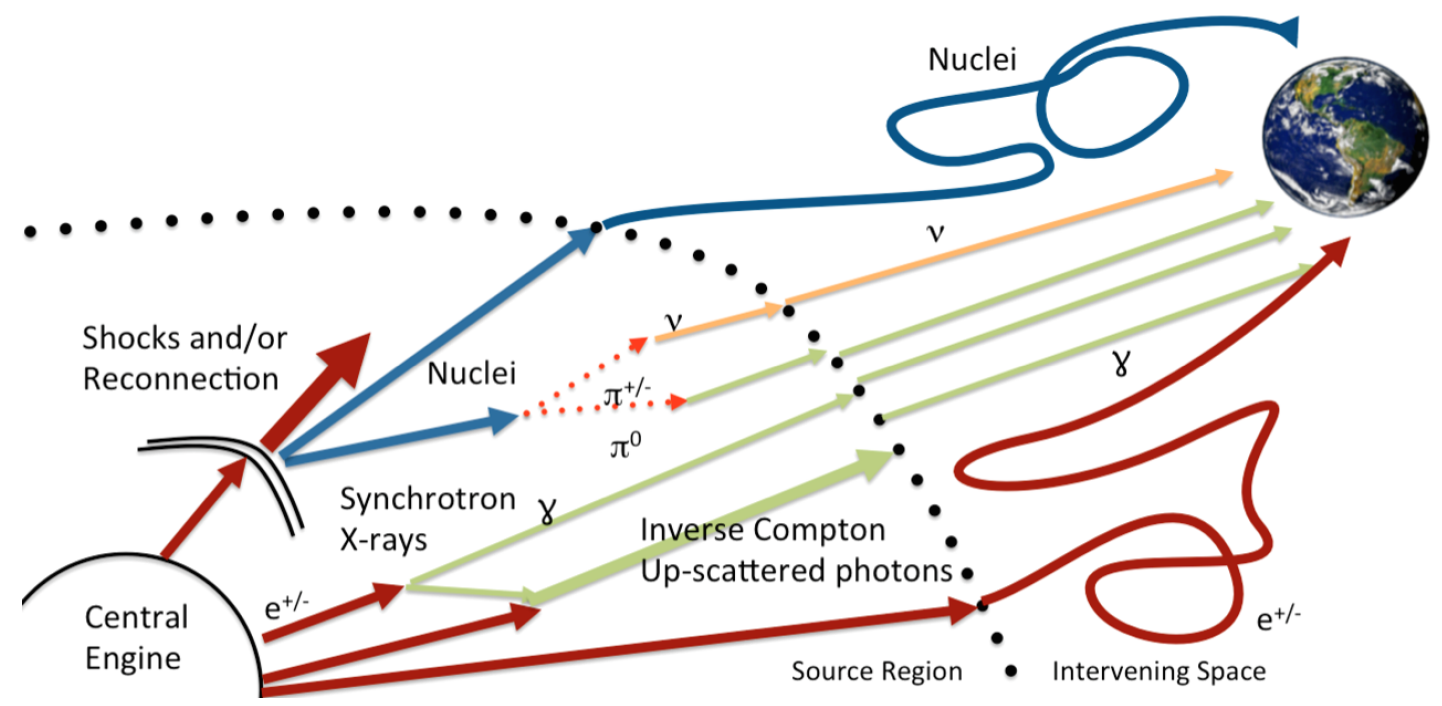

Figure 1: A schematic overview of the standard picture of a cosmic accelerator. High-energy electrons and hadronic cosmic-rays are accelerated in a source. The messengers received at Earth can be either these charge particles directly propagating from the source, or neutral secondaries (photons or neutrinos) produced in pion decay or inverse Compton scattering within the source.

\section{Introduction}

The High Altitude Water Cherenkov Observatory (HAWC) was completed in early 2015 and is currently surveying the Northern sky for gamma-ray sources between $100 \mathrm{GeV}$ and $100 \mathrm{TeV}$. HAWC is distinctive for its sensitivity to extreme high-energy photons, wide field-of-view and ability to operate continuously. In one year, HAWC will survey the entire overhead sky above $\sim 5$ $\mathrm{TeV}$ with the sensitivity [1] of a 50-hour pointed observation by the current generation of Imaging Atmospheric Cherenkov Telescopes.

The central objective of HAWC is to study particle acceleration in high-energy sources and subsequent propagation of these particles to Earth. Figure 1 shows a schematic of the standard picture of particle acceleration in sources. The chief complication in modeling these sources is whether hadronic cosmic rays or electrons and positrons are responsible for the observed gamma rays. HAWC's measurements can illuminate the nature of these accelerators by characterizing the highest energy these sources can achieve, the long-duration time structure of the high-energy emission, triggering follow-up by partner observatories, and by measuring the extended emission as the high-energy particles stream from their sources.

The HAWC science goals include:

- Distinguishing gamma-ray emission caused by electron acceleration or hadronic cosmic-ray acceleration is a persistent challenge in modeling accelerators. In order to build a complete picture of a source, it is critical to know the highest energy particles a source can accelerate. HAWC has unprecedented sensitivity above $10 \mathrm{TeV}$ and will be used to measure gamma-ray spectra of Galactic sources up to $100 \mathrm{TeV}$. 
- The rapid variability of some sources points to acceleration in regions that are too small to be spatially resolved by gamma-ray instruments. With HAWC, we continuously monitor the sky for bright $\sim \mathrm{TeV}$ outbursts from Active Galaxies, Gamma-Ray Bursts or Galactic transients, enabling long-duration $\mathrm{TeV}$ light curves and multi-wavelength followup and study.

- A number of large gamma-ray structures have been seen in the Galaxy, including extended emission in the Galactic plane, most notably in the Cygnus arm of the Galaxy, in the region around the Geminga pulsar, and the two extended lobes of gamma-rays (the Fermi Bubbles). With HAWC, we have an unbiased survey of the Northern sky, and a full accounting of all the $\mathrm{TeV}$ photons in the sky.

- HAWC will search for a number of signatures of new physics, including Dark Matter annihilation, Lorentz Invariance Violation and Primordial Black Hole evaporation.

- Hadronic cosmic-rays at Earth constitute the chief background to gamma-ray observation. Nevertheless, HAWC data can be used to study the population of $100 \mathrm{GeV}-100 \mathrm{TeV}$ hadronic cosmic rays directly, and can use the cosmic rays as a probe of solar physics, through the sun's modulation of the cosmic rays at Earth. Spatial anisotropies of cosmicrays are not well understood and may probe local magnetic field structure.

Section 2 discusses the state of the HAWC detector. HAWC scientific efforts are divided into four categories described in the following sections: In Section 3, we present results on gamma-ray studies of objects in the Galaxy. Section 4 discusses gamma-ray observations of objects outside our galaxy. Section 5 presents our efforts to constrain fundamental physics using astrophysical gammaray observations. Section 6 discusses our efforts to study the cosmic-rays directly, including the impact of the sun on the cosmic-ray population.

\section{HAWC Instrument}

Figure 2 shows the completed HAWC detector at the 4100 meter altitude site near Pico de Orizaba in Mexico. The HAWC instrument consists of an array of 300 water Cherenkov detectors (WCDs). Each WCD comprises a steel tank 7.3 meters in diameter and 4.5 meter height, a plastic bladder to contain 188,000 liters of purified water, and four PMTs: three 8-inch Hamamatsu R5912 PMTs re-used from Milagro and one 10-inch R7081-MOD high-quantum efficiency PMT. The WCDs are deployed in a close-packed array over an area of approximately 20,000 $\mathrm{m}^{2}$. Cables connect the PMTs to a central counting house, deliver high voltage to the PMTs, and carry signals to the acquisition electronics. A calibration system [2] allows the delivery of light via optical cable to the WCDs in order to measure the length of the cables and the relationship between the amount of light delivered and the measured signal in PMTs.

The PMTs detect Cherenkov light from energetic particles passing through the WCDs during cosmic-ray and gamma-ray air showers. The air shower reconstruction takes place in three steps: First, the air shower core, the dense concentration of energetic particles directly along the primary particle's trajectory, is found with a fit to the energy density recorded in PMTs on the ground. Second, the air shower direction is determined. Air showers propagate near the speed of light, so 


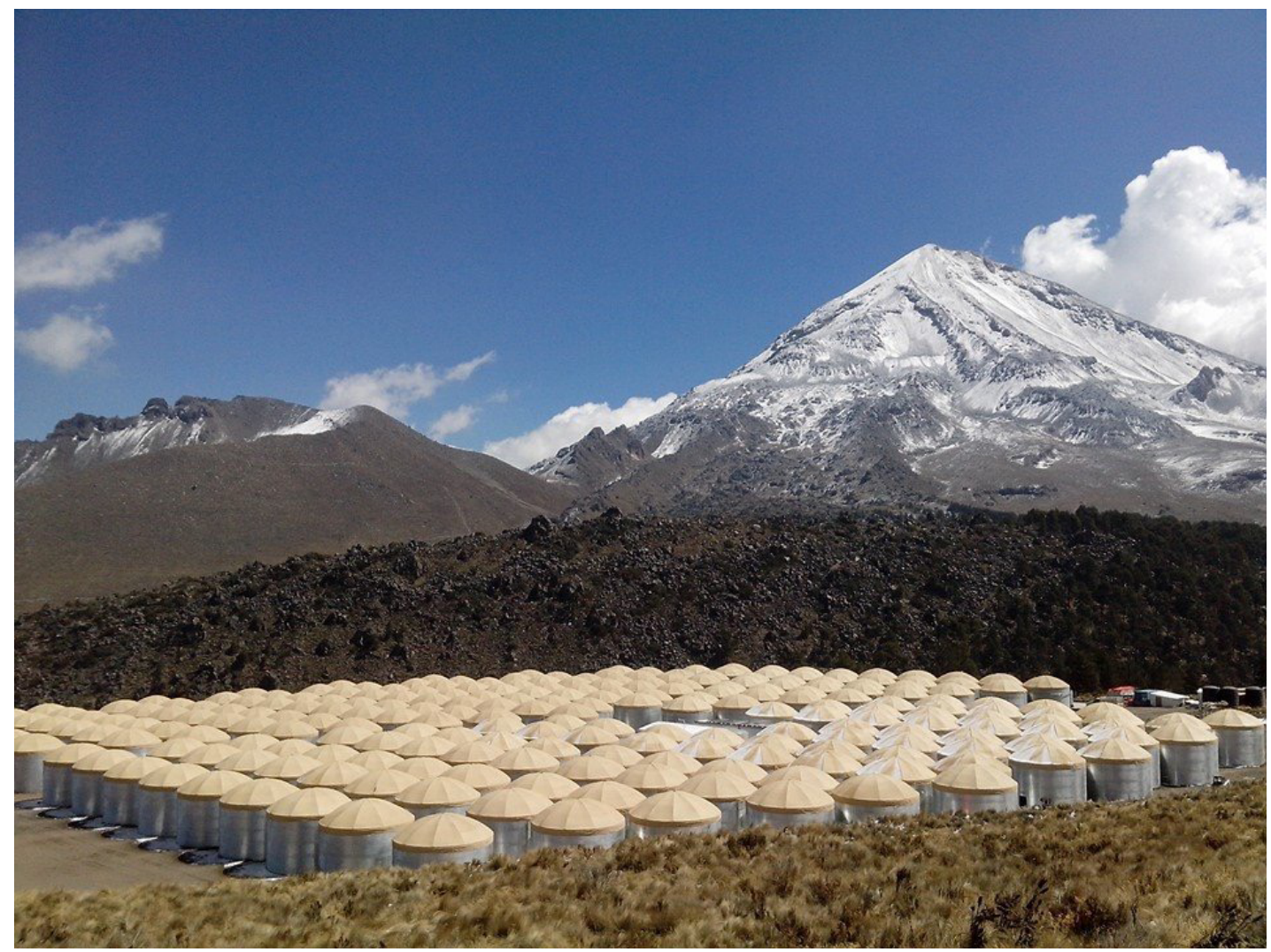

Figure 2: The High Altitude Water Cherenkov Observatory at its home near Pico de Orizaba in Mexico. The $300 \mathrm{WCDs}$ are large and close-packed giving the instrument a footprint of $20,000 \mathrm{~m}^{2}$.

the energetic particles arrive nearly in a plane, with particles far from the core arriving somewhat delayed. The PMT hit times are fit to this hypothesis to determine the shower direction. In a final step, a number of event parameters are computed to determine if the event is likely to be a photonor hadron-initiated particle cascade.

Figure 3 shows two events recorded in HAWC. In both cases, the air shower core is readily discernible as well as the planar time distribution. The hadron-initiated event in the left panel has a number of isolated regions of high-energy particles well outside the core region. The gamma-ray event in the right panel is much more smooth, with no large charge deposition outside the shower core region. These characteristics form the basis for the current photon/hadron discrimination in HAWC [3], and additional advanced separation techniques are being investigated [4, 5].

The chief limitation of HAWC's sensitivity at energies above about $10 \mathrm{TeV}$ is the collection area of the instrument. To first order, air shower particles arrive in a plane defined by the speed of light, but particles far from the shower axis arrive delayed. The effect is substantial, up to tens of ns 100 meters from the air shower core. The accurate reconstruction of showers requires correction of this effect, and the correction is done imperfectly if the air shower core is well off the main instrumented area. Off-instrument events are usable. The separation of photons and hadrons can be done, but without a reliable core localization, the direction cannot be accurately determined. In order to recover these events, a new sparse outrigger array is planned [6]. Such an array will, at 

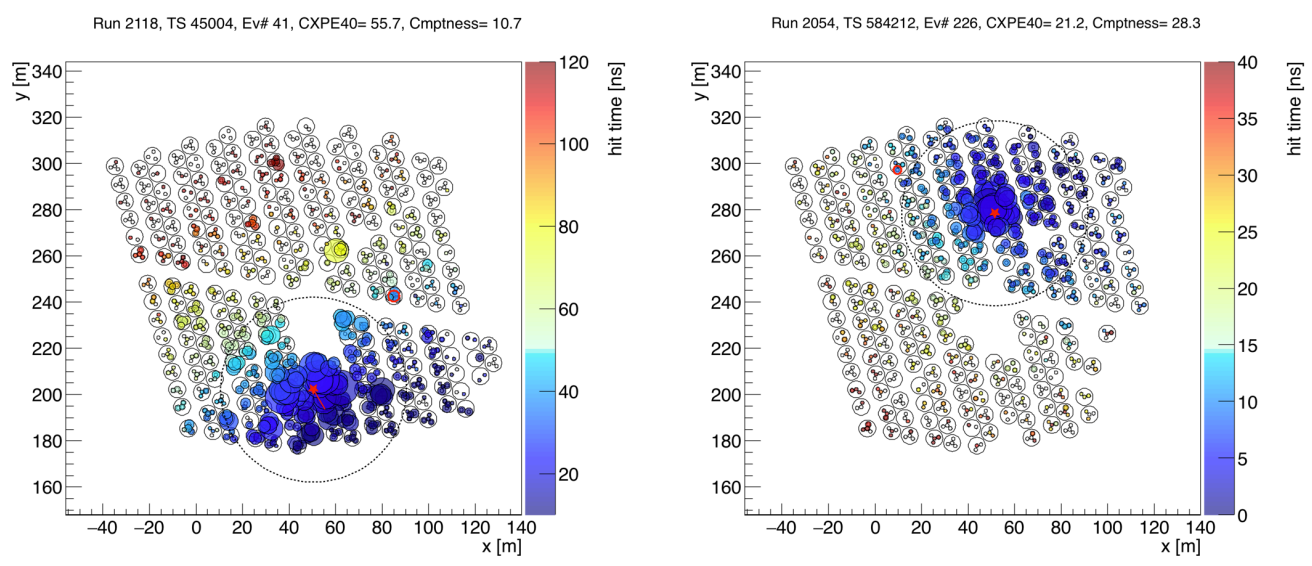

Figure 3: Two events recorded in HAWC. Each point represents a PMT in HAWC and the WCD tank boundaries are shown. The color indicates the time each PMT saw light and the the size indicates how much light was seen. In each case, the shower core is readily apparent as the dense region of hard-hit PMTs. The left event is a hadronic cosmic-ray event, with a number of regions of isolated energy deposition outside the shower core. The right figure came from the direction of the Crab nebula and is likely to be a true $\sim 10 \mathrm{TeV}$ photon. Note the absence of energy deposition far from the core for the photon, denoted by the 40-meter circle drawn around the core.

modest additional cost, greatly enhance the high-energy sensitivity of HAWC.

After air showers have been reconstructed, we make a map of the sky. Figure 4 shows a significance map from the first 150 days of the near-final HAWC instrument in Galactic coordinates. Clear emission along the plane of the galaxy is seen, along with emission from the Crab Nebula, and the TeV blazars Markarian 421 and 501.

This paper presents data from two datasets:

- HAWC-111: Prior to the completion of HAWC, the instrument was operated in a partial configuration. HAWC-111 refers to the instrument between August 2, 2013 and July 7, 2014, during which between 106 and 133 of the total 300 WCDs were operational, for a total livetime of 283 days.

- HAWC: HAWC began operating in its near-final configuration with 247 of its 300 WCDs live on November 26, 2014. We present data from 149 live days.

The integral sensitivity of the full HAWC sample is almost double the sensitivity of the HAWC-111 sample, but the understanding of the HAWC-111, and the evaluation of its systematics, is more mature. Some analyses are presented from the HAWC-111 epoch and some from the full HAWC operation.

\section{Galactic Science}

HAWC data clearly shows $\mathrm{TeV}$ emission along the Galactic plane including a number of sources and extended regions of emission. 


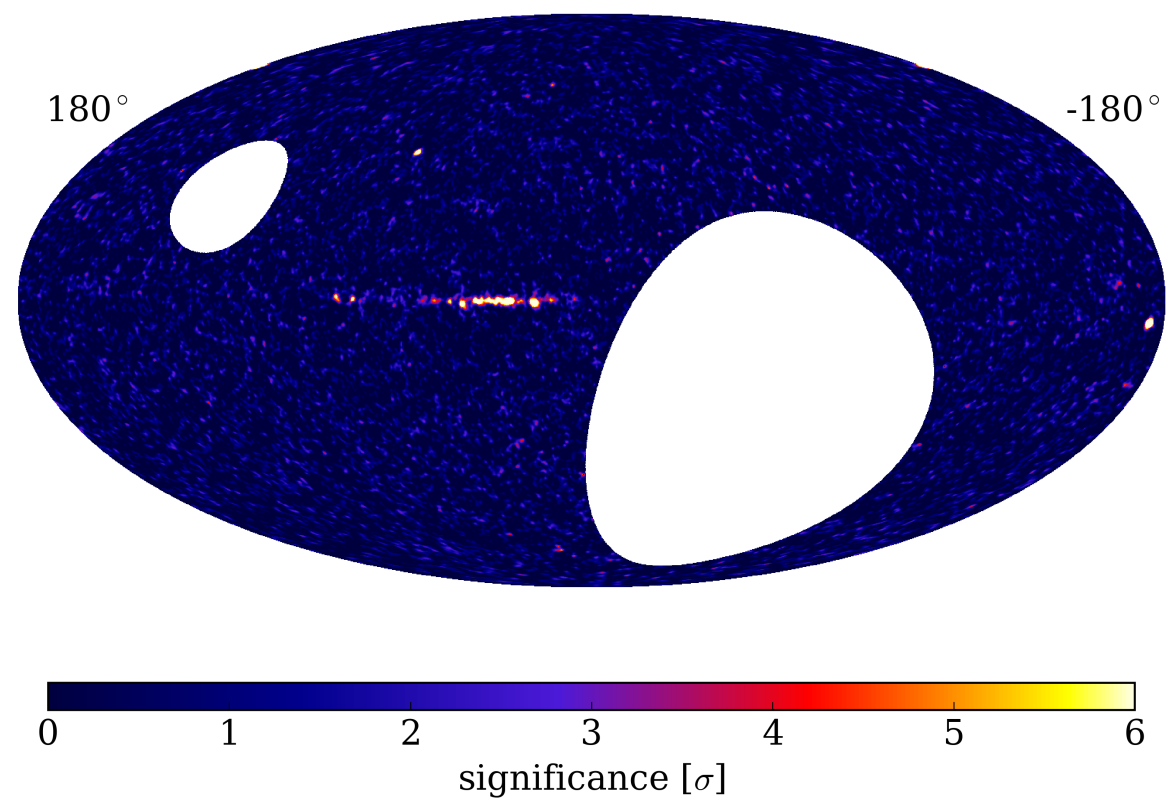

Figure 4: The all-sky skymap from 150 days of the nearly-completed HAWC instrument. The sky is shown in Galactic coordinates. TeV emission from a number of source regions in the Galactic plane are evident. The Crab is seen to the far right. The TeV blazars Markarian 501 and 421 are detected at high significance. Markarian 501 is conspicuously located above the plane of the galaxy. Markarian 421, in this projection, is right at the edge of the field, near the top.

The Crab Nebula, the standard candle in $\mathrm{TeV}$ astronomy, has been observed at high confidence [7]. With the instrument in its near-final configuration, we are observing the Crab at more than $3 \sigma$ each day, for a total significance of $\sim 38 \sigma$ in the first 150 days of data.

The Crab detection is particularly important because it allows us to validate the modeling and performance of the instrument. In particular, we are able to generate an extremely pure photon sample and can study the instrument response to photons of known direction. Figure 5 shows the field-of-view of the Crab for extremely large events (the easiest to distinguish from hadronic background). After photon/hadron cuts, we have a signal to background ratio greater than 10 . This is unprecedented for a wide-field ground array. The strong hadron rejection in the vicinity of the Crab has been used to place an upper limit on isotropic photon emission above $10 \mathrm{TeV}$. [8]. Currently, HAWC data is within a factor of 2 of the design sensitivity and is improving with our understanding of the instrument.

Figure 6 shows a region of the Galactic plane visible to HAWC. The data is shown from 283 days of data in the HAWC-111 configuration. An analysis has been developed [9] which allows us to model the region as an ensemble of point sources [10]. This results in the detection of 10 sources and source candidates [11, 12], most of which are associated with known TeVCat [13] sources. Searches for Galactic transients $[14,15]$ are in progress.

In addition to the region of the inner Galactic plane, HAWC data also reveals a region of extended emission around the Geminga pulsar [16]. Geminga is nearby (about $250 \mathrm{pc}$ ) and has the 

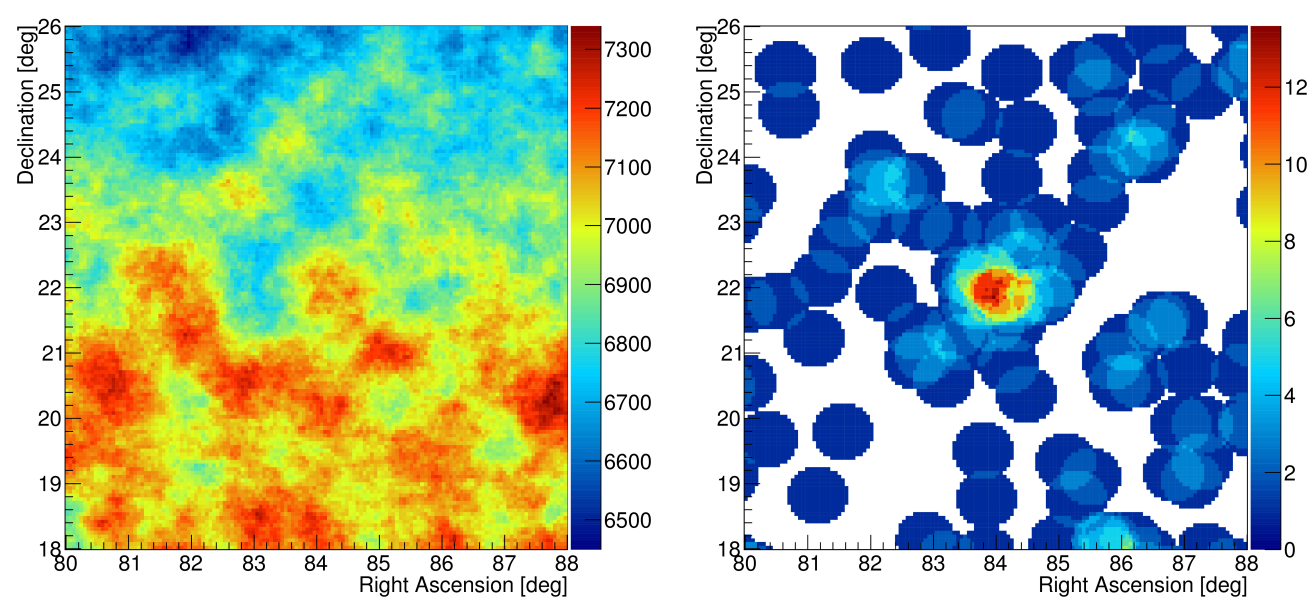

Figure 5: Skymap in equatorial coordinates in the vicinity of the Crab Nebula in 105 days of data with HAWC-250 (shown in the J2014/15 epoch) from [8]. Only events with more than $85 \%$ of the available PMTs are shown. The color scale shows the number of events detected within a $0.45^{\circ}$ circle around each point in the sky. The left figure shows the region with no cuts and is completely dominated by hadronic cosmic rays. The slight drift in rate is due to the changing acceptance of the detector, located at $+19^{\circ} \mathrm{N}$ latitude, for events further from zenith. The figure on the right shows the same skymap after a strong cut which removes all but 1 in $\sim 10^{4}$ background events. After cuts, at the location of the Crab, we observe 12 events with an expected background of $0.81 \pm 0.05$ in this sample.

potential to explain $\sim \mathrm{GeV}$ positron excess [17] as direct production of electrons and positrons [18]. $\mathrm{TeV}$ emission around Geminga was first observed with Milagro [19], but the source's large extent has defied identification by TeV IACTs. In HAWC data, the source appears very large. More data is required to fit an extent with reliable errors, but a $3^{\circ}$ round bin maximizes the statistical significance of the detection at $6.3 \sigma$ pre-trials.

The analysis of the excess around Geminga is in progress, but the source, at a declination of $\sim 18^{\circ}$, is near the Crab Nebula. This proximity permits direct comparison to the Crab, with the most relevant systematic issues canceling. Figure 8 shows the number of excess events in a large $3^{\circ}$ region around Geminga as a function of the size of the events in HAWC, relative to the same analysis applied to the Crab. Larger events are higher energy with "Bin 0" events just below $1 \mathrm{TeV}$ and "Bin 9" events at $\sim 10 \mathrm{TeV}$. The total number of photons from Geminga is large, approximately $40 \%$ of the total brightness of the Crab. If the Geminga data were flat in this figure, we would conclude it had the same spectral properties as the Crab. The slight trend upward, while not significant, suggests a harder spectrum than the Crab. It is worth noting a hard spectrum was predicted for Geminga in [18]. A more complete treatment, and more exposure is necessary, though, to measure the spectrum accurately.

Finally, HAWC has a unique capability to search for large, extended $\mathrm{TeV}$ emission from the Fermi Bubbles [20], two large gamma-ray structures extending above the center of the Galaxy. HAWC-111 data has been searched and upper limits have been placed [21]. Figure 9 shows the preliminary limits on the North Fermi Bubble. HAWC limits rule out a pure $\mathrm{E}^{-2}$ continuation of the spectrum. 

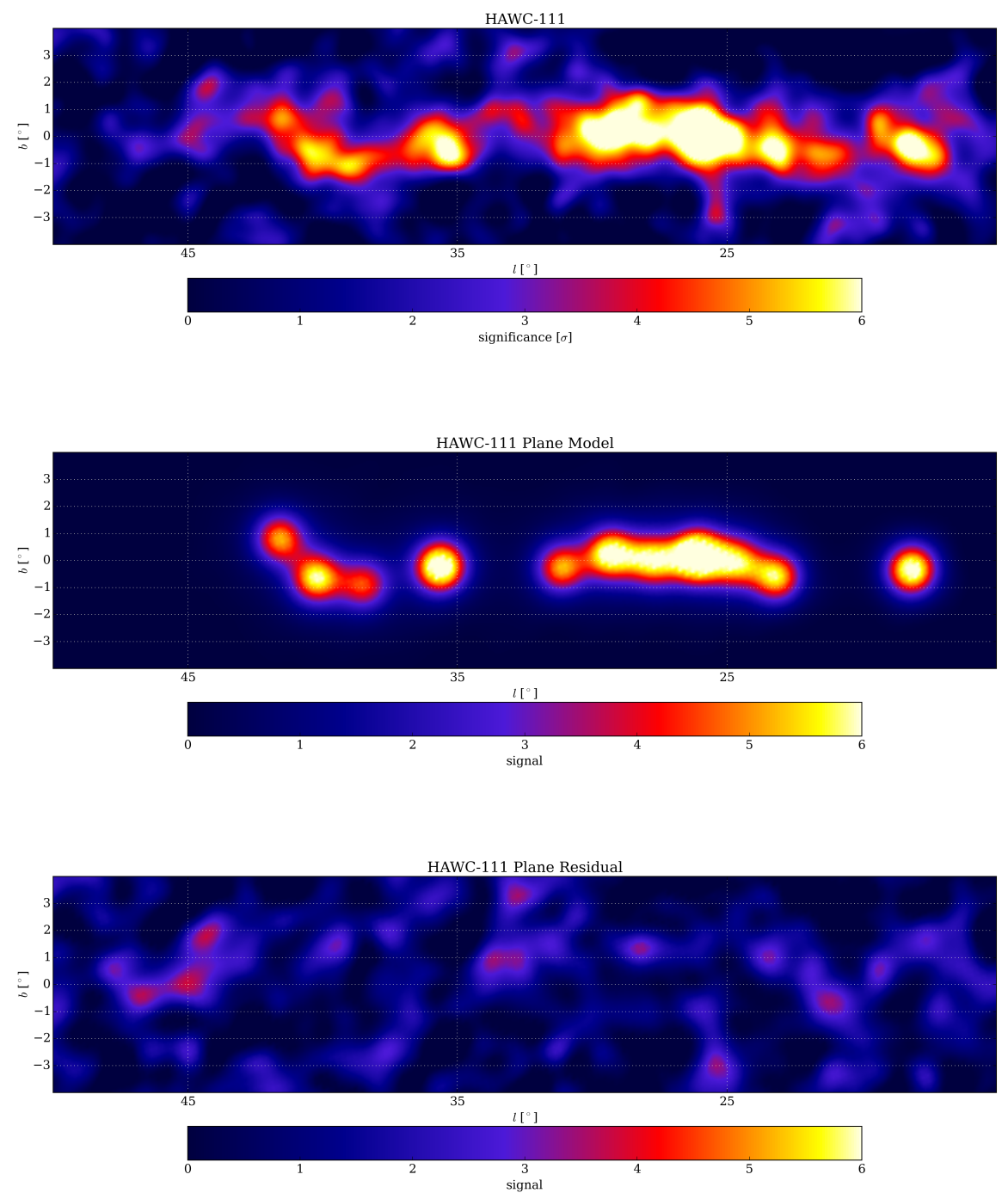

Figure 6: The Galactic plane survey in HAWC-111 data [12, 11]. The top figure shows the significance map of the inner region of the Galactic plane. The middle figure shows the 11-source model developed which explains the emission, resulting in 10 sources and source candidates. The bottom figure shows the residual emission, showing that all significant point sources have been successfully removed.

\section{Extra-Galactic Science}

$\mathrm{TeV}$ photons produced outside our Galaxy may be attenuated by pair production on the ExtraGalactic Background Light (EBL) that fills the space between galaxies. HAWC, with sensitivity to photons above $100 \mathrm{GeV}$, maintains sensitivity to Extra-Galactic objects that are nearby and bright, most notably Active Galactic Nuclei (AGN) and Gamma-Ray Bursts (GRBs). With HAWC, we monitor the sky for transient emission from these objects. This monitoring is being conducted in real-time at the HAWC site [23, 24].

Archival searches are also underway. In archival HAWC data, the blazars Markarian 421 

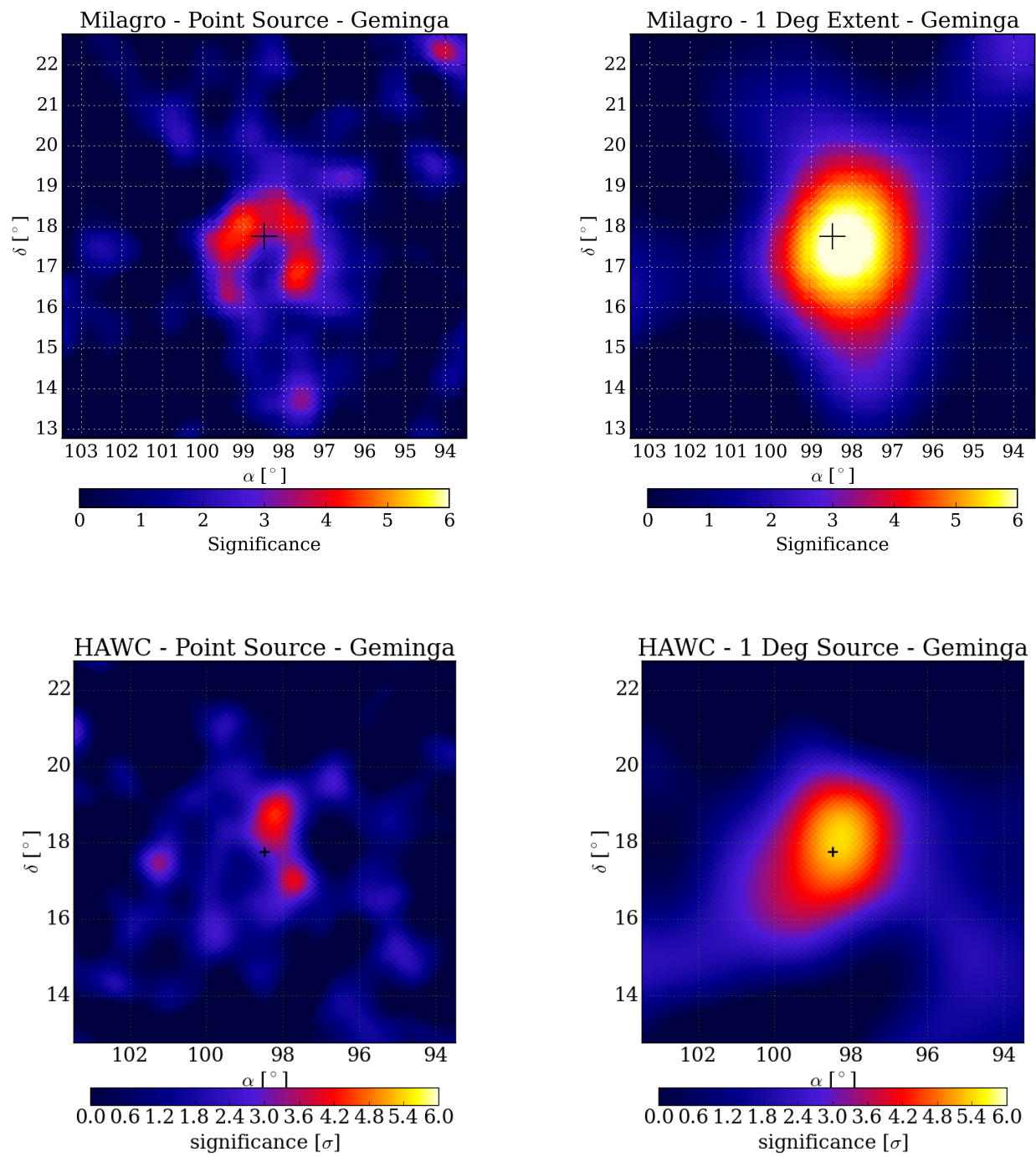

Figure 7: HAWC and Milagro observation of Geminga [16]. The region of TeV emission around Geminga is large and extended. The top (bottom) leftmost figure shows the Milagro (HAWC) statistical significance map, assuming the source is a point source. The region is much more significant under the assumption that the source is extended by 1 degree, shown on the right.

and 501 show substantial, significant flux variability [25]. Figure 10 shows an example from this analysis, the long-term light curve of Markarian 421, in data from HAWC-111, binned in weeklong time bins. During the HAWC observation, the blazar showed significant, substantial flux variability. Analysis is underway.

In addition to AGN, HAWC has the sensitivity to observe a nearby bright Gamma-Ray Burst if it is in the field-of-view of the instrument. Fermi observations show a number of bursts (e.g. [27]) with a high-energy spectral component which is rising up to the high-energy limits of Fermi, where the HAWC sensitive energy begins. One estimate [28], extrapolating GRB populations observed in Fermi, suggests HAWC might optimistically observe 1-2 GRBs per year. 


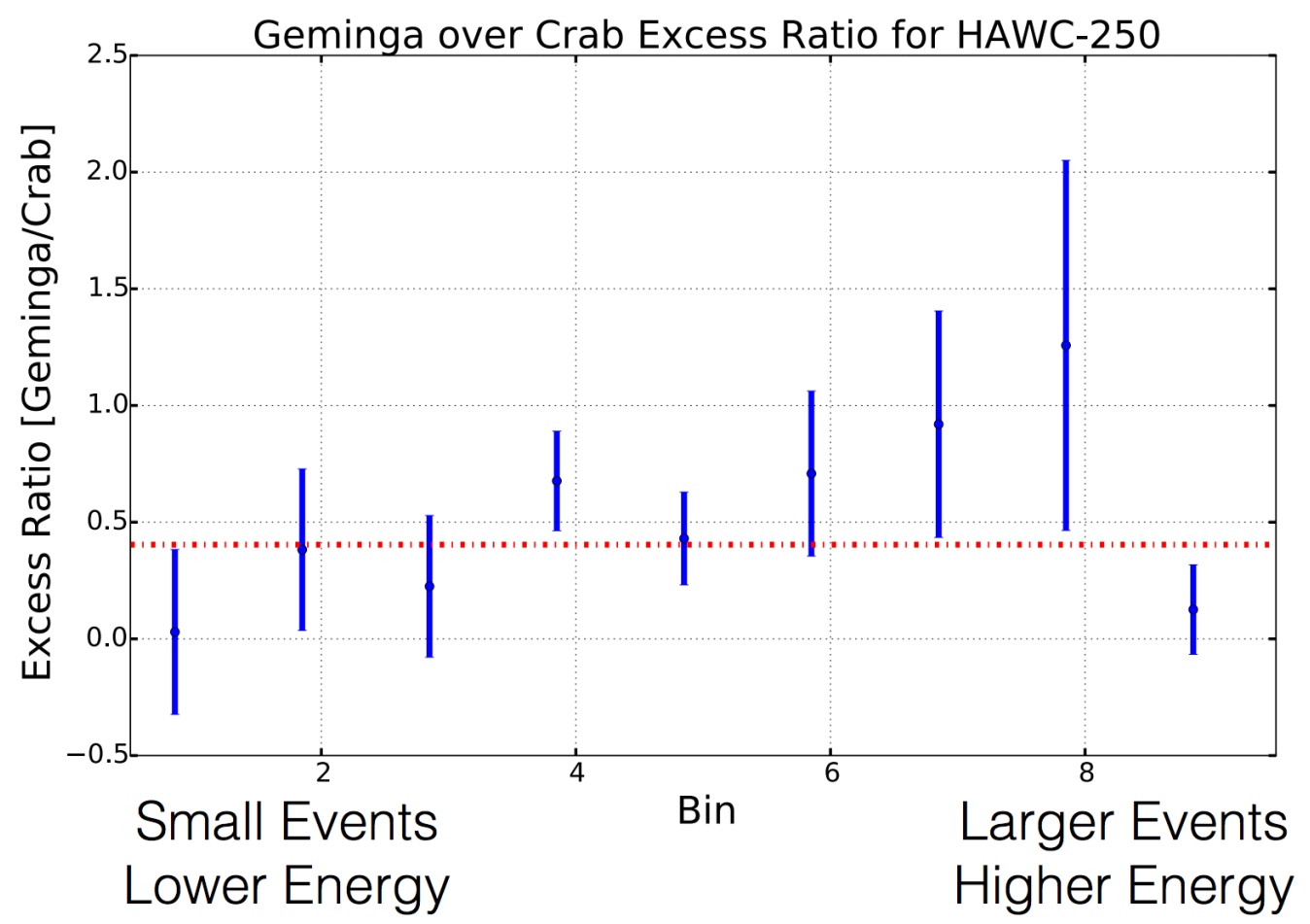

Figure 8: The relative gamma-ray excess from the Geminga and the Crab in data from the nearly-completed HAWC detector [16]. The excess is shown as a function of "analysis bin" which is a proxy for the energy of gamma-ray events. The gentle upward trend suggests that the source region may be harder than the Crab with a total surface brightness approximately $40 \%$ of the Crab.

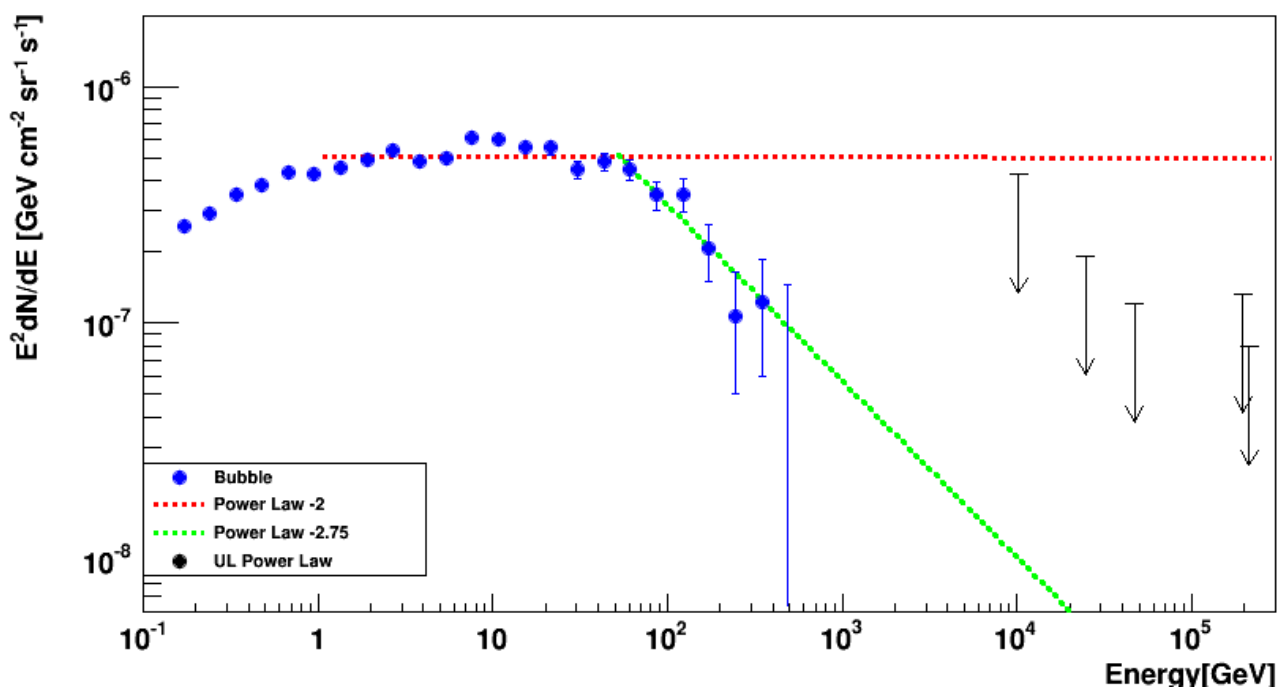

Figure 9: Preliminary upper limits on the North Fermi Bubble with HAWC-111 data [21]. The Bubble flux from [22] is shown along with two extrapolations, assuming it continues at $\mathrm{E}^{-2}$ or $\mathrm{E}^{-2.75}$. Preliminary HAWC upper limits are shown in black and rule out a pure $\mathrm{E}^{-2}$ continuation of the Fermi Bubbles. 


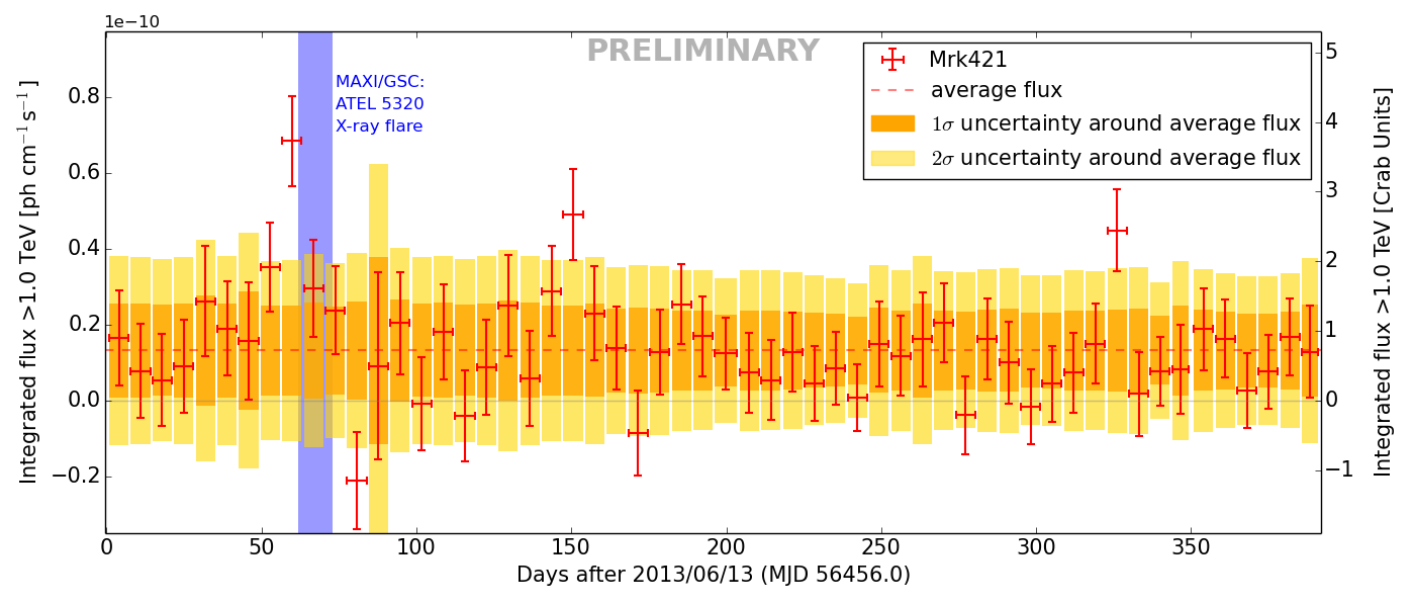

Figure 10: Flux light curve of Markarian 421 for the time between June 13, 2013, and July 9, 2014, in intervals of 7 days, with horizontal error bars indicating the time span between the start of the first and the end of the last transit over HAWC [25]. Integrated fluxes above $1 \mathrm{TeV}$ for a differential spectrum with power law index 2.2 and exponential cut-off at $5 \mathrm{TeV}$ are shown in photons per $\mathrm{cm}^{2}$ per second on the left axis and divided by the average Crab flux observed by HAWC on the right axis. The statistical 1 and $2 \sigma$ flux uncertainties of the individual measurements are displayed as orange/yellow bars around the weighted average flux (dashed line). The large uncertainties in the flux around day $\sim 90$ are due to a period of construction and maintenance in September 2013 during which HAWC was shut down during day time for several days in a row, creating large gaps in the transit coverage. The blue band indicates the period over which a strong increase in X-ray flux was observed by MAXI [26].

During HAWC-111, 22 Swift-detected GRBs occurred in the HAWC field-of-view, and we have data on 18 of them. HAWC data was searched [29] for evidence of temporally coincident bursts of events. Observations were consistent with background. Figure 11 shows the significance distribution of the 18 GRBs in HAWC data along with random "fake" triggers, distributed as expected, to validate the null hypothesis determination.

\section{Fundamental Physics}

Understanding the particle nature of dark matter is one of the most pervasive questions in physics. If dark matter consists of multi-TeV particles that can annihilate (predicted by many WIMP models), we expect some level of gamma-ray production in dense regions of dark matter: the Galactic Center, dark matter satellites of our galaxy, or nearby galaxies or galaxy clusters. Because HAWC has a wide field-of-view, we can search every candidate in our field-of-view.

Figure 12 shows the results of a search for a dark matter signal from dwarf galaxies in the field-of-view of HAWC using 180 days of the HAWC-111 dataset. The dwarf galaxies have been individually searched for a photon excess, and combined into a global upper limit [30]. Eventually HAWC limits will be competitive at extremely high masses above 10-1000 TeV [31]. HAWC will also be particularly sensitive to decaying dark matter and dark matter in galaxy clusters, where the sources are expected to be more extended. 


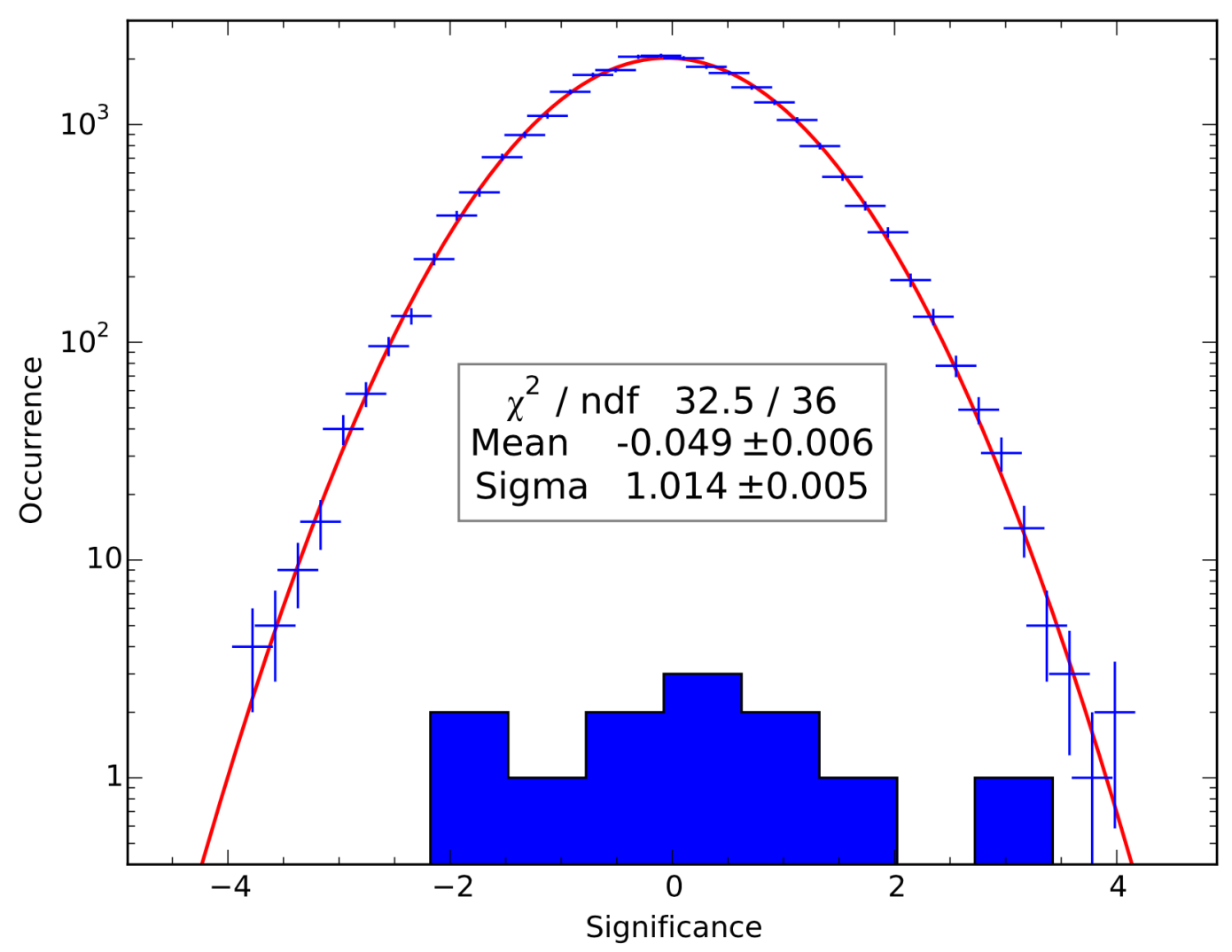

Figure 11: The significance distribution of 18 Swift-triggered GRBs as seen in HAWC (shaded blue) [29]. All events are consistent with pure background. Additionally, the significance distribution of a large number of injected triggers is also shown, validating the computation of the statistical significance.

HAWC is uniquely suited to search for bursts of $\mathrm{TeV}$ photons from primordial black hole (PBH) evaporation [32, 33]. The photon emission from PBHs is broad-band [34] and may be difficult to distinguish from a Gamma-Ray Burst. If a PBH evaporation event does occur, they must be in the vicinity of the Earth where the EBL attenuation of $\mathrm{TeV}$ photons will not be present, as it is for GRBs. As such, if HAWC sees multi-TeV photons of the correct spectral and temporal shape, we can positively identify an evaporation event.

A suitable GRB, at sufficiently high redshift and with a sufficiently hard spectrum, if observed by HAWC, could be used to derive limits on Lorentz Invariance Violation: energy-dependent variations of the speed of light. HAWC's higher reach in energy, compared to satellite-based instruments, provides it with the ability to set competitive limits [35].

\section{Cosmic-Ray Science}

Finally, HAWC serves as a unique observatory for studying cosmic rays that arrive at Earth, studying what is normally regarded as our background to gamma-ray observation. In particular, HAWC triggers at $\sim 15 \mathrm{kHz}$ and will collect more than a trillion cosmic rays between $100 \mathrm{GeV}$ and $100 \mathrm{TeV}$ before the experiment is finished. In one second, we resolve the relative cosmic-ray flux 


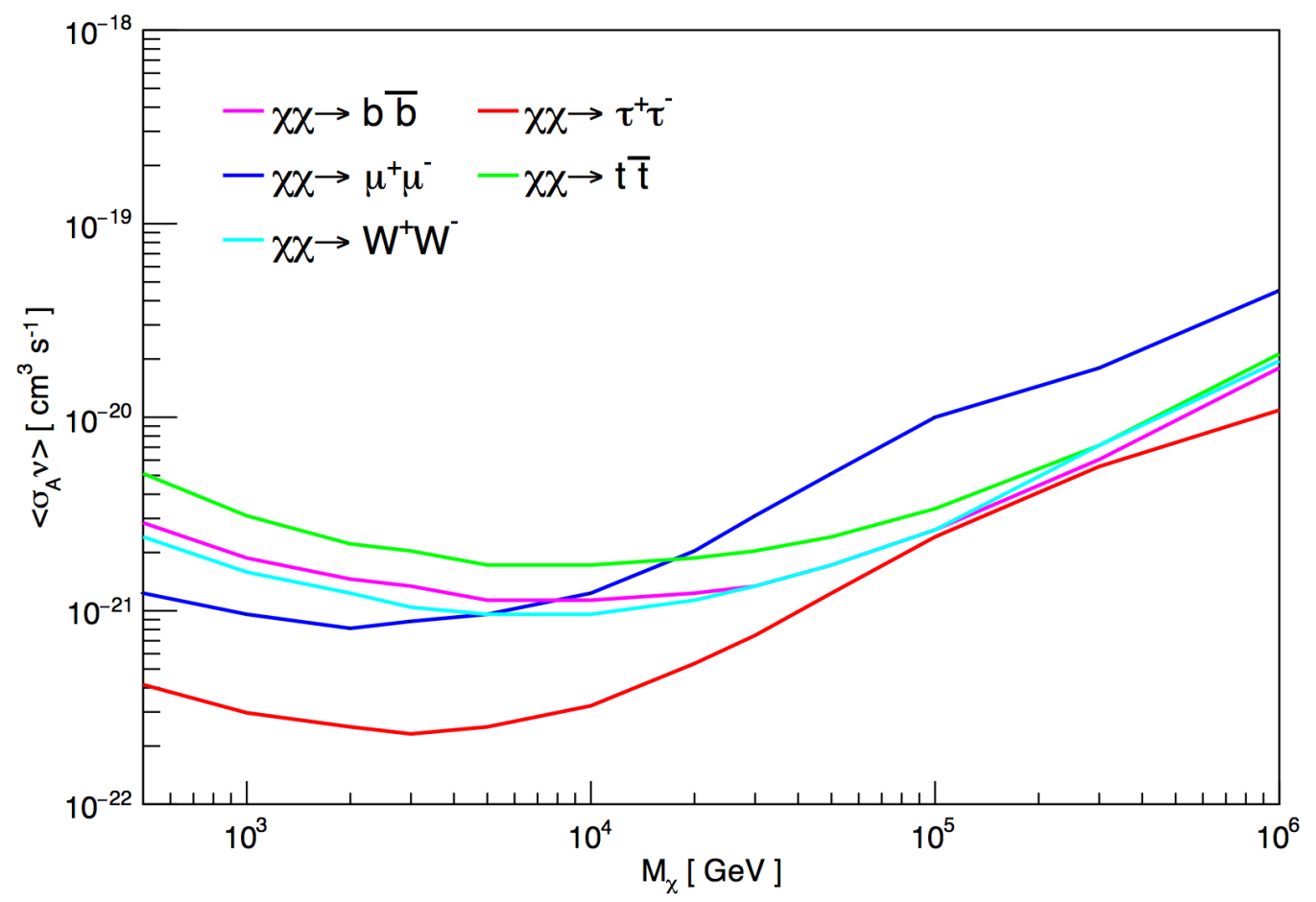

Figure 12: Combined annihilation cross-section limits from HAWC's non-observation of photons of 14 dwarf spheroidal galaxies [30]. The velocity-weighted cross section limit is shown versus the mass of the dark matter particle mass. The limit depends on the assumed annihilation products.

to about $1 \%$, and minute fluctuations in the cosmic-ray flux, either due to solar modulation [36] or due to spatial fluctuations in the cosmic-ray background [37] can be seen.

Figure 13 shows the HAWC view of the $10^{\circ}$-scale cosmic-ray anisotropy first discovered in Milagro data [38]. These anisotropies are small, a few parts in $10^{4}$. HAWC data confirms the Milagro detections: "Region A" and "Region B", and adds to it a third detected excess termed "Region C" first reported in ARGO-YBJ data [39]. With increasing sensitivity, it is becoming possible to investigate the spectral properties of the anisotropy [37] and to cross-correlate with anisotropy seen by IceCube cosmic-ray observations in the south [40].

HAWC is such a precise cosmic-ray barometer that it is possible to study the local cosmicray modulation by the sun [36] and to image the cosmic-ray silhouette of the sun [41], allowing a time-dependent picture of the magnetic field of the sun.

Finally, HAWC's photon/hadron separation admits the possibility to study cosmic-ray electrons [42]. Cosmic-ray electrons will induce pure electro-magnetic showers, just like photons, but will appear as a large diffuse population, not localized like photons.

\section{Summary and Outlook}

HAWC brings new capabilities to $\mathrm{TeV}$ astronomy and astrophysics. With its wide field-ofview and continuous operation, HAWC is poised to discover new sources and continuously monitor the sky for rare transient events across the Northern sky. Interestingly enough, the Southern sky 


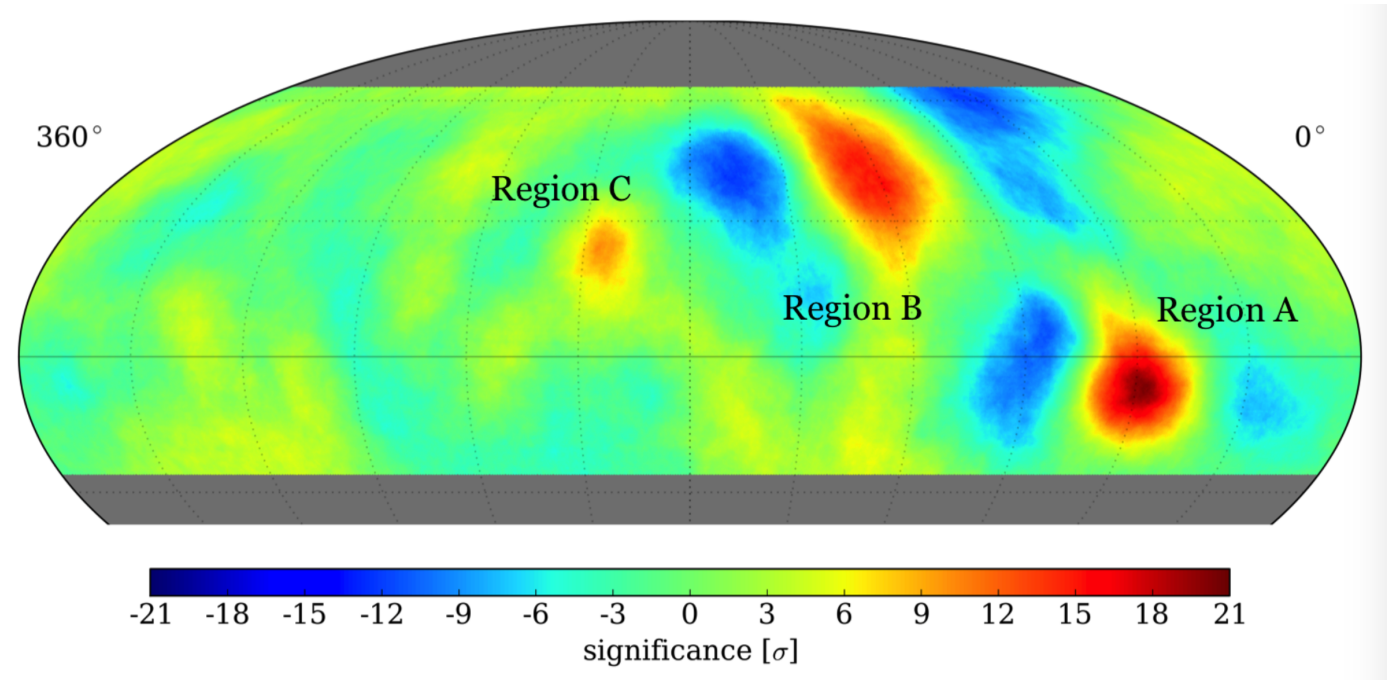

Figure 13: The HAWC cosmic-ray skymap, using data from HAWC-111, in equatorial coordinates [37]. The map has had large-scale dipole, quadrupole and octopole components subtracted, and a $10^{\circ}$ smoothing applied. Milagro’s Regions A and B are seen, along with a new Region C.

has never been surveyed with a wide-field $\mathrm{TeV}$ instrument. Such an instrument [43] is under consideration. With HAWC we will continuously survey the sky for the highest-energy photons ever detected and are poised to revolutionize the field of high-energy astrophysics.

\section{Acknowledgments}

We acknowledge the support from: the US National Science Foundation (NSF); the US Department of Energy Office of High-Energy Physics; the Laboratory Directed Research and Development (LDRD) program of Los Alamos National Laboratory; Consejo Nacional de Ciencia y Tecnología (CONACyT), Mexico (grants 260378, 55155, 105666, 122331, 132197, 167281, 167733); Red de Física de Altas Energías, Mexico; DGAPA-UNAM (grants IG100414-3, IN108713, IN121309, IN115409, IN111315); VIEP-BUAP (grant 161-EXC-2011); the University of Wisconsin Alumni Research Foundation; the Institute of Geophysics, Planetary Physics, and Signatures at Los Alamos National Laboratory; the Luc Binette Foundation UNAM Postdoctoral Fellowship program.

\section{References}

[1] A. U. Abeysekara et al., Sensitivity of the high altitude water Cherenkov detector to sources of multi-TeV gamma rays, Astroparticle Physics 50 (Dec., 2013) 26-32, [arXiv: 1306.5800 ].

[2] H. A. Ayala Solares et al., The Calibration System of the HAWC Gamma-Ray Observatory, International Cosmic Ray Conference (2015) [arXiv: 1508.04312 ].

[3] A. J. Smith et al., HAWC Design, Operation, Reconstruction and Analysis, International Cosmic Ray Conference (2015) [arXiv:1508.05826].

[4] Z. Hampel et al., Gamma Hadron Separation using Pairwise Compactness Method with HAWC, International Cosmic Ray Conference (2015) [arXiv: 1508.04047 ].

[5] T. Capistrán, I. Torres, L. Altamirano, et al., New Method for Gamma/Hadron separation in HAWC using Neural Networks, International Cosmic Ray Conference (2015) [arXiv: 1508 . 04370]. 
[6] A. Sandoval et al., HAWC Upgrade with a Sparse Outrigger Array, International Cosmic Ray Conference (2015) [arXiv:1509.04269].

[7] F. Salesa Greus et al., Observations of the Crab Nebula with Early HAWC Data, International Cosmic Ray Conference (2015) [arXiv: 1508.04274$].$

[8] J. Pretz et al., Limit on an Isotropic Diffuse Gamma-Ray Population with HAWC, International Cosmic Ray Conference (2015) [arXiv:1508.04091].

[9] P. W. Younk et al., A High-Level Analysis Framework for HAWC, International Cosmic Ray Conference (2015) [arXiv:1508.07479].

[10] A. U. Abeysekara et al., Search for TeV Gamma-Ray Emission from Point-like Sources in the Inner Galactic Plane with a Partial Configuration of the HAWC Observatory, arXiv: 1509.05401. Submitted to the Astrophysical Journal.

[11] C. M. Hui, H. Zhou, et al., HAWC Observation of Supernova Remnants and Pulsar Wind Nebulae, International Cosmic Ray Conference (2015) [arXiv: 1508.07391].

[12] H. Zhou, C. M. Hui, P. Hüntemeyer, et al., TeV Observations of the Galactic Plane with HAWC and Joint Analysis of GeV Data from Fermi, International Cosmic Ray Conference (2015) [arXiv:1509.02138].

[13] http://tevcat.uchicago.edu.

[14] S. BenZvi et al., Looking for Gamma-ray Emission from TeV Binary Candidates with HAWC, International Cosmic Ray Conference (2015) [arXiv: 1508 .03589].

[15] C. Alvarez Ochoa et al., Searching for Very High Energy Emission from Pulsars Using the High Altitude Water Cherenkov (HAWC) Observatory, International Cosmic Ray Conference (2015) [1508.05008].

[16] B. Baughman, J. Wood, et al., TeV Gamma-Ray Emission Observed from Geminga with HAWC, International Cosmic Ray Conference (2015) [arXiv: 1508 .03497].

[17] O. Adriani et al., An anomalous positron abundance in cosmic rays with energies 1.5-100GeV, Nature 458 (Apr., 2009) 607-609, [arXiv:0 810 . 4995].

[18] H. Yüksel, M. D. Kistler, and T. Stanev, TeV Gamma Rays from Geminga and the Origin of the GeV Positron Excess, Physical Review Letters 103 (July, 2009) 051101, [arXiv: 0810 . 2784].

[19] A. A. Abdo et al., Milagro Observations of Multi-TeV Emission from Galactic Sources in the Fermi Bright Source List, ApJ 700 (Aug., 2009) L127-L131, [arXiv: 0904 . 1018].

[20] M. Su, T. R. Slatyer, and D. P. Finkbeiner, Giant Gamma-ray Bubbles from Fermi-LAT: Active Galactic Nucleus Activity or Bipolar Galactic Wind?, ApJ 724 (Dec., 2010) 1044-1082, [arXiv:1005.5480].

[21] H. A. Ayala Solares, C. M. Hui, P. Hüntemeyer, et al., Fermi Bubbles with HAWC, International Cosmic Ray Conference (2015) [arXiv:1508.06592].

[22] M. Ackermann et al., The Spectrum and Morphology of the Fermi Bubbles, ApJ 793 (Sept., 2014) 64, [arXiv:1407.7905].

[23] T. Weisgarber, I. G. Wisher, et al., Blazar Alerts with the HAWC Online Flare Monitor, International Cosmic Ray Conference (2015) [arXiv:1508.05399].

[24] J. Wood et al., All-sky sensitivity of HAWC to Gamma-Ray Bursts, International Cosmic Ray Conference (2015) [arXiv:1508.04120]. 
[25] R. J. Lauer, P. W. Younk, et al., Results from monitoring TeV blazars with HAWC, International Cosmic Ray Conference (2015) [arXiv:1508.04479].

[26] S. Ueno et al., MAXI/GSC detection of a bright X-ray flare from Mrk 421, The Astronomer's Telegram 5320 (Aug., 2013) 1.

[27] M. Ackermann et al., Fermi Observations of GRB 090510: A Short-Hard Gamma-ray Burst with an Additional, Hard Power-law Component from $10 \mathrm{keV}$ TO GeV Energies, ApJ 716 (June, 2010) 1178-1190, [arXiv:1005.2141].

[28] I. Taboada and R. C. Gilmore, Prospects for the detection of GRBs with HAWC, Nuclear Instruments and Methods in Physics Research A 742 (Apr., 2014) 276-277, [arXiv:1306.1127].

[29] D. Lennarz, I. Taboada, et al., First Results from HAWC on GRBs, International Cosmic Ray Conference (2015) [arXiv: 1508.07325].

[30] M. Longo Proper, B. Dingus, and J. P. Harding, First Limits on the Dark Matter Cross-Section with the HAWC Observatory, International Cosmic Ray Conference (2015) [arXiv: 1508.04470].

[31] J. P. Harding, B. Dingus, et al., Dark Matter Annihilation and Decay Searches with the High Altitude Water Cherenkov (HAWC) Observatory, International Cosmic Ray Conference (2015) [arXiv:1508.04352].

[32] T. Ukwatta et al., Sensitivity of HAWC to Primordial Black Hole Bursts, International Cosmic Ray Conference (2015) [arXiv:1508.03573].

[33] A. A. Abdo, Milagro limits and HAWC sensitivity for the rate-density of evaporating Primordial Black Holes, Astroparticle Physics 64 (Apr., 2015) 4-12, [arXiv: 1407.1686 ].

[34] T. Ukwatta et al., Observational Characteristics of the Final Stages of Evaporating Primordial Black Holes, International Cosmic Ray Conference (2015) [arXiv: 1507.01648 ].

[35] L. Nellen et al., The potential of the HAWC Observatory to observe violations of Lorentz Invariance, International Cosmic Ray Conference (2015) [arXiv: 1508 .03930].

[36] O. Enríquez, A. Lara, R. Caballero-Lopez, et al., Solar Event Simulations in the HAWC Scaler System, International Cosmic Ray Conference (2015) [arXiv: 1508 .07285].

[37] S. BenZvi, D. Fiorino, S. Westerhoff, et al., Observation of Anisotropy in the Arrival Direction Distribution of TeV Cosmic Rays with HAWC, International Cosmic Ray Conference (2015) [arXiv:1508.04781].

[38] A. A. Abdo et al., Discovery of Localized Regions of Excess 10-TeV Cosmic Rays, Physical Review Letters 101 (Nov., 2008) 221101, [arXiv: 0801 .3827].

[39] B. Bartoli et al., Medium scale anisotropy in the TeV cosmic ray flux observed by ARGO-YBJ, Phys. Rev. D 88 (Oct., 2013) 082001, [arXiv:1309.6182].

[40] J. C. Díaz-Vélez, D. Fiorino, et al., Full-Sky Analysis of Cosmic-Ray Anisotropy with IceCube and HAWC, International Cosmic Ray Conference (2015).

[41] O. Enríquez, A. Lara, et al., The Galactic Cosmic-Ray Sun Shadow observed by HAWC, International Cosmic Ray Conference (2015) [arXiv:1508.07351].

[42] S. BenZvi, M. Un Nisa, Z. Hampel, et al., Measuring the $e^{+} e^{-}$Flux Above 1 TeV with HAWC, International Cosmic Ray Conference (2015) [arXiv:1306.1508.].

[43] M. DuVernois et al., Detector Considerations for a HAWC Southern Observatory, International Cosmic Ray Conference (2015) [arXiv: 1508.03669 ]. 